\title{
The Effect of Visual Feedback on One-hand Gesture Performance in Vision-based Gesture Recognition System
}

\author{
Junho Kim ${ }^{1}$, Ji Hyoun Lim , Sung Hyun Moon ${ }^{2}$ \\ ${ }^{1}$ Department of Industrial Engineering, Hongik University, Seoul, 121-791 \\ ${ }^{2}$ Department of Computer Science, Seoul National University, Seoul, 151-742
}

\begin{abstract}
Objective: This study presents the effect of visual feedback on one-hand gesture performance in vision-based gesture recognition system when people use gestures to control a screen device remotely. Backgroud: gesture interaction receives growing attention because it uses advanced sensor technology and it allows users natural interaction using their own body motion. In generating motion, visual feedback has been to considered critical factor affect speed and accuracy. Method: three types of visual feedback(arrow, star, and animation) were selected and 20 gestures were listed. 12 participants perform each 20 gestures while given 3 types of visual feedback in turn. Results: People made longer hand trace and take longer time to make a gesture when they were given arrow shape feedback than star-shape feedback. The animation type feedback was most preferred. Conclusion: The type of visual feedback showed statistically significant effect on the length of hand trace, elapsed time, and speed of motion in performing a gesture. Application: This study could be applied to any device that needs visual feedback for device control. A big feedback generate shorter length of motion trace, less time, faster than smaller one when people performs gestures to control a device. So the big size of visual feedback would be recommended for a situation requiring fast actions. On the other hand, the smaller visual feedback would be recommended for a situation requiring elaborated actions.
\end{abstract}

Keywords: Visual feedback, One-hand gesture, Vision-based gesture recognition system

\section{Introduction}

제스처 인터렉션은 비언어적 상호작용의 한 종류로서, 사 용자의 신체를 직접적으로 사용하여 기계나 시스템을 조작 하는 형태이다. 이러한 제스처 인터렉션은 인간의 동작을 상호작용의 기반으로 하고 있어서, 동작 즉 제스처에 의미 를 부여하는 방식(Kuhnel et al., 2011; Nebelrath et al., 2011), 동작의 편의성(Stern, Wachs, \& Edan, 2008), 인식 시스템의 특성 등 다양한 측면의 연구 주제를 내포하고 있다 (Do et al., 2006; Lee et al., 2010; Chang et al., 2011).
제스처 인터랙션 관련하여 최근에는 사용자 중심적 관점 의 중요성이 두드러지면서, 동작 인식 등을 사용한 제어 상 황에 관한 연구가 많이 진행되고 있다(Lim et al., 2012). 특히 동작 인식과 관련된 기존 연구들은 동작을 이용하여 제 어하는 상황과 관련하여, 동작 인식 분야에서 인간의 요인을 고려하여 시스템의 성능을 향상시키려는 연구나(Stern et al., 2008), 상황의 속성, 사용자의 속성, 동작의 물리적 속성 등을 통해 동작의 적합성을 알아내는(Kuhnel et al., 2011; Nebelrath et al., 2011) 등 제스처에 사용되는 동작 자체에 관한 연구들이 보고되고 있다.

한편, 동작 인식 시스템과 관련해서는 동작 인식에 쓰이는

Corresponding Author: Ji Hyoun Lim. Department of Industrial Engineering, Hongik University, Seoul, 121-791.

Phone: +82-2-320-3072, E-mail: limjh@hongik.ac.kr

Copyright@2012 by Ergonomics Society of Korea(pISSN:1229-1684 eISSN:2093-8462). All right reserved.

(c) This is an open-access article distributed under the terms of the Creative Commons Attribution Non-Commercial License(http://creativecommons.org/licenses/by-nc/3.0/), which permits unrestricted non-commercial use, distribution, and reproduction in any medium, provided the original work is properly cited. http://www.esk.or.kr 
센서와 알고리즘에 따라 사용 가능한 동작의 종류와 범위가 달라진다(Do et al., 2006; Lee et al., 2010; Chang et al., 2011). 또한 접촉식 제스처 인터랙션, 즉 터치 인터랙션과 관련한 연구들을 살펴보면, 터치 스크린에 제시되는 시각 자 극의 특성 (크기, 위치 등)에 따라 터치 영역이나 조작행동에 변화가 관찰된 바 있다(Park et al., 2010; Sun et al., 2007; Parhi et al., 2006). 접촉식 제스처 관련하여서는 스크린 상 에 나타나는 시각정보의 레이아웃, 메뉴 접근성 등의 효과를 관찰(Balagtas-Fernandex et al., 2009)하거나, 시각 자극 의 모양, 위치, 배경과의 색대비를 바꿀 때 사람의 누르는 지 점의 편향도와 반응 시간을 살펴본 연구( Lim et al., 2011) 가 보고된 바 있다.

제스처 기반의 인터랙션은 자연스러운 동작을 기반으로 인간과 기계가 상호작용하는 방식이기 때문에, 사용자에게 사용자의 신체의 움직임에 대하여 어떤 방식의 feedback을 제공하느냐에 따라 사용자의 움직임 자체가 영향을 받을 수 도 있다고 가정할 수 있다. 본 연구에서는 동작 인식을 사용 한 원거리 조작 상황에서 디스플레이 상에 나타나는 visual feedback의 종류에 따라서 사용자의 동작 수행이 어떻게 달 라지는지 알아보고자 한다.

\section{Method}

본 연구에서 사용한 동작 인식 시스템은 손끝의 궤적을 추 적하여 그 동선을 파악하는 방식이다. 동작 인식 시스템은 Kinect와 프로젝터, 노트북으로 구성되었다. 손 끝 위치에 대한 visual feedback은 화살표, 애니메이션, 별 형태를 사 용하였다. 화살표 형태의 visual feedback은 이 실험에서 선정한 3 가지 visual feedback type 중 가장 크기가 작고, windows system에서 커서로 사용되는 화살표 모양을 사 용하였다. 애니메이션 형태 visual feedback은 화살표에 비해 1.5 배의 크기이다. 애니메이션의 특징은 손의 위치에 별이 나타나고, 손이 움직이면 그 궤적을 따라 작은 별들이 나타났다 시차를 두고 사라지는 형태이다. 별 형태 visual feedback은 화살표의 2.5배 크기이다. Figure 1에서 세 종 류의 visual feedback을 확인할 수 있다.

이와 같이 3 가지 종류의 visual feedback에 대해서 각각 20 가지 제스처를 수행하였다. 20가지의 제스처는 손 끝이 그리는 모양을 인지하는 방식인 edge 제스처에 해당하는 것들 위주로 선정하였다. 그 각각에 대한 형태와 예시는 Figure 2와 같다. 여기에서 명시된 3가지 visual feedback 과 20가지 제스처 각각에 대하여 사용자의 제스처의 수행 차이를 실험적으로 검증하여 보았다.
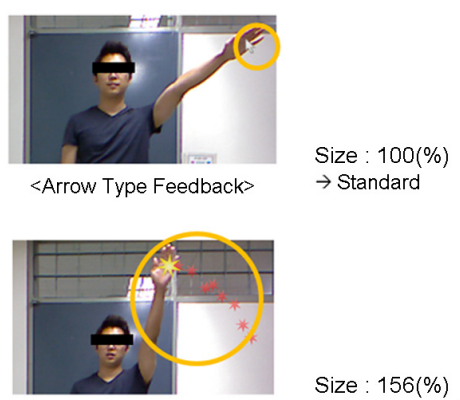

<Animation Type Feedback>

Size : $156(\%)$

$\rightarrow$ Comparing Arrow Feedback Type Size

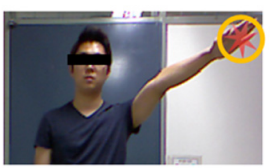

<Star Type Feedback $>$

Size : $250(\%)$

$\rightarrow$ Comparing Arrow Feedback Type Size

- Size of each feedback type is defined as the distance between the points which are farhest to each other in the figure

- Size of animation type feedback is determined by the size of the biggest figure

Figure 1. Three types of visual feedback
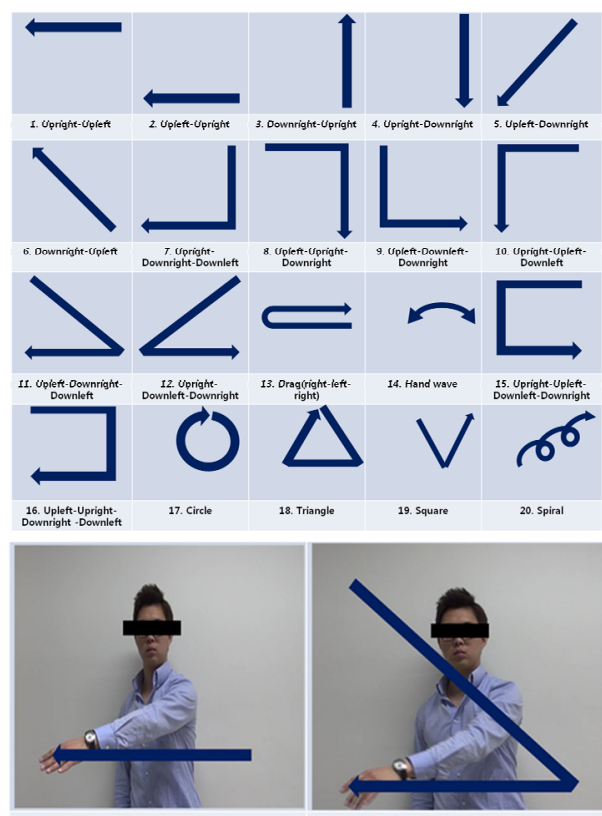

2. Downright-Downleft

11. Upleft-Downright-Downleft

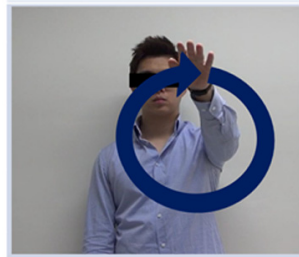

17. Circle

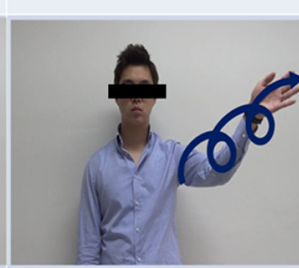

20. Spiral
Figure 2. Gesture list and captured image demonstrating the gestures 


\subsection{Experiment design}

본 실험의 목적은 동작 인식을 사용한 원거리 조작 상황에 서 디스플레이 상에 나타나는 피드백의 종류에 따라서 사용 자의 동작 수행의 차이를 실험적으로 검증하는 것이다. 실험 에서의 독립 변수는 3 가지 visual feedback type이며, 독립 변수는 세 수준(화살표, 별, 애니메이션 형태)을 가진다. 종 속 변수는 제스처 수행을 정량적으로 측정한 값으로, 해당 제스처를 수행하는데 걸린 시간, 제스처 수행 시 손이 움 직인 거리, 제스처 수행 속도이다. 본 실험의 실험 가설은 'display 상의 visual feedback 방식이 달라짐에 따라 사용 자의 제스처 수행 특성이 달라질 것이다이고, 그 상세한 내 용은 다음과 같다. 여기서 수행 특성은 종속 변수 세 가지로 설명할 것이다.

\subsection{Participants \& apparatus}

남성 7 명, 여성 5 명 (총 12 명)의 실험참여자가 본 실험에 참여하였으며, 평균 연령은 25.1세였다. 이 12명의 실험참 여자 가운데 오른손잡이 11 명, 왼손잡이는 1 명 이었다.

제스처 수행에 대한 데이터를 수집하기 위한 프로그램은 $\mathrm{C \#}$ 으로 작성되었고, Kinect로 인식한 피실험자의 모습과 손 의 위치는 $1,280 \times 1,024$ 해상도로 전면에 제시하였다. 피실 험자들이 프로젝터를 통해 본인의 행동과 손의 움직임을 볼 수 있다. 실험이 진행된 실험 환경의 모습은 Figure 3 과 같다.

실험 자극은 앞서 정의된 제스처 20가지에 대하여 동영 상을 제작하여 사용하였다. 동영상 일부를 캡쳐한 장면이 Figure 2에 제시되어 있다. 대부분의 피실험자들이 오른손잡 이인 것을 고려하여 영상을 보고 제스처를 따라 하는 것이 용이하도록 거울상인 왼손으로 촬영한 영상을 사용하였다.

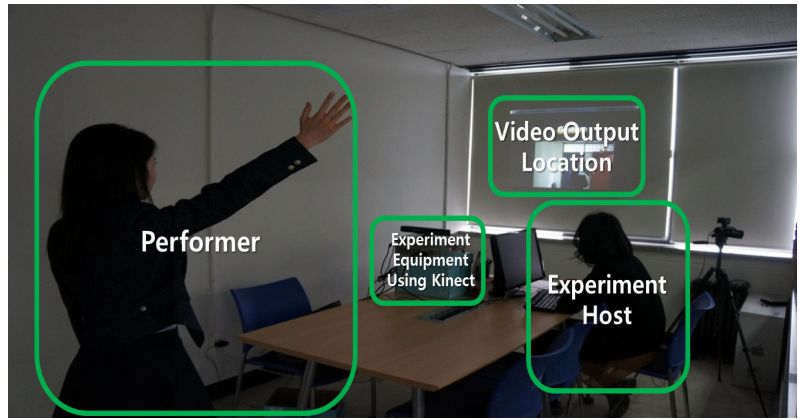

Figure 3. Experiment set up

\subsection{Experiment procedure}

실험절차는 실험 소개, 동작 습득, 본 실험, 실험 후 제스 처에 대한 의견 조사로 진행되었다. 실험 소개 단계에서는 실험의 목적과 방법에 대해 실험참여자에게 설명하고, 동작 데이터 수집에 대한 동의를 구하였다. 본 실험에서는 먼저 세 종류의 visual feedback을 순서를 무작위로 정한 후, 정 해진 순서에 따라 실험을 진행하였다. 그 후 randomize된 순서대로 나오는 20 가지 제스처에 해당하는 영상을 피실험 자에게 Tablet PC를 사용하여 보여준다. 추가 요청이 있는 경우 다시 보여주었다. Figure 1에서 보듯이, 화면 위 쪽에 제스처 번호가 제시되면 동작을 수행한다. 이와 같이 한 동 작에 대해 3 번 반복하여 제스처를 수행하고, 수행에 대한 데이터가 수집되었다. 한 종류의 visual feedback에 대하여 20가지 제스처에 대한 수행이 모두 끝나면 잠시 휴식 후, 정해진 순서대로 visual feedback에 대한 실험을 실시한다. 본 실험이 모두 끝나면 피실험자를 대상으로 수행했던 3가 지 visual feedback에 대한 선호도에 대한 주관적 평가를 수집하였다.

\section{Results}

3 가지 visual feedback type에 대하여 수행한 피실험자 12 명의 데이터를 취합하여 각각의 동작을 거리, 시간, 속도 데이터로 결과를 정리하였다. 거리, 시간, 속도 데이터는 각 각 평균, 표준편차로 표현된다. 정리된 데이터의 내용은 Table 1 과 같다. 이 데이터를 토대로, 피실험자들의 visual feedback에 따른 제스처 수행 차이를 제스처 수행 시 손

Table 1. Summary of performance data

\begin{tabular}{|c|c|c|c|}
\hline & \multicolumn{3}{|c|}{ Visual feedback } \\
\hline Trace length(m) & Arrow & Star & Animation \\
\hline Average & 3.8916 & 3.8322 & 3.8855 \\
\hline Standard deviation & 0.7856 & 0.7746 & 0.7614 \\
\hline Elapsed time(s) & Arrow & Star & Animation \\
\hline Average & 3.7154 & 3.5045 & 3.6265 \\
\hline Standard deviation & 0.7667 & 0.7283 & 0.7609 \\
\hline Motion speed(m/s) & Arrow & Star & Animation \\
\hline Average & 1.0833 & 1.1319 & 1.107 \\
\hline Standard deviation & 0.2942 & 0.3025 & .2727 \\
\hline
\end{tabular}


이 움직인 거리(trace length), 제스처 수행 시간(elapsed time), 제스처 수행 속도(motion speed)로 나누어 파악할 수 있다. 또한 이를 통해 visual feedback의 특성과 제스처 수행 특성과의 관계를 알아낼 수 있다. 본 실험이 모두 끝나 고 피실험자에게서 3가지 feedback에 대한 선호도 조사를 실시하였다. 점수를 차등 배분하여 1 순위에게 3 점, 2 순위에 게 2점, 3 순위에 3 점을 주어 계산을 하였다. 선호도 조사 결 과는 Table 2에 요약되어 있다. 선호도 점수 계산 결과 피 실험자들은 애니메이션 형태 feedback을 가장 많이 선호하 였고(32점), 그 다음으로는 별 형태 feedback을 선호하였 고(22점), 화살표 형태 feedback을 가장 덜 선호하였다 (18점).

Table 2. Subjective preference on the feedback type

\begin{tabular}{|c|c|c|c|c|c|c|}
\hline & \multicolumn{3}{|c|}{ Preference(Ranking) } & \multicolumn{3}{|c|}{ Preference(Scoring) } \\
\hline & Arrow & Star & Animation & Arrow & Star & Animation \\
\hline 1 & 3 & 2 & 1 & 1 & 2 & 3 \\
\hline 2 & 2 & 1 & 3 & 2 & 3 & 1 \\
\hline 3 & 2 & 1 & 3 & 2 & 3 & 1 \\
\hline 4 & 3 & 2 & 1 & 1 & 2 & 3 \\
\hline 5 & 3 & 1 & 2 & 1 & 3 & 2 \\
\hline 6 & 3 & 2 & 1 & 1 & 2 & 3 \\
\hline 7 & 2 & 3 & 1 & 2 & 1 & 3 \\
\hline 8 & 3 & 2 & 1 & 1 & 2 & 3 \\
\hline 9 & 3 & 2 & 1 & 1 & 2 & 3 \\
\hline 10 & 3 & 2 & 1 & 1 & 2 & 3 \\
\hline 11 & 2 & 3 & 1 & 2 & 1 & 3 \\
\hline 12 & 3 & 1 & 2 & 1 & 3 & 2 \\
\hline \multicolumn{4}{|c|}{ Sum } & 18 & 22 & 32 \\
\hline
\end{tabular}

\section{Analysis}

각각의 visual feedback에 따라 피실험자들의 제스처 수 행 시 손이 움직인 거리(trace length), 제스처 수행 시간 (elapsed time), 제스처 수행 속도(motion speed)에 차이 가 있는지는 visual feedback 요인에 대한 분산분석을 통 해 검증해 보았고, 이후 각 조건 간의 차이는 쌍체 비교 (paired t-test)를 통하여 살펴보았다. 분산분석 결과는 Table 3 에 요약되어 있다.
Table 3. ANOVA for types of visual feedback

\begin{tabular}{c|c|c|c}
\hline & df & $F$-value & $p$-value \\
\hline Time & 2 & 14.27 & $<\mathbf{0 . 0 0 1}$ \\
\hline Distance & 2 & 1.27 & 0.28 \\
\hline Speed & 2 & 5.05 & $<\mathbf{0 . 0 0 1}$ \\
\hline
\end{tabular}

\subsection{Elapsed time of performing gesture}

3가지의 visual feedback 형태(별, 애니메이션, 화살표) 에 따라 제스처 수행 시간에 차이가 있는지를 검증하기 위 해서 각 visual feedback 별로 제스처 20개에 대한 반복 수 3 번 총 60 회의 수행을 12 명의 실험참여자가 실시하여, 총 720 개의 데이터가 수집되었다. 이 데이터를 사용하여 세 집단 간 분산분석을 실시하였다. 분산분석 결과 visual feedback 종류에 따라서 제스처 수행 시간의 차이는 통계적 으로 유의한 것으로 판명이 되었다 $(p<0.001)$. 좀 더 구체적 으로 각각의 visual feedback 간 수행 시간 차이를 알아보 기 위해 별 형태 feedback, 애니메이션 형태 feedback, 화 살표 형태 feedback를 2개씩 짝을 지어 쌍체 비교 (paired t-test)를 실시하였다.

Table 4에서 보듯이 쌍체 비교의 결과, 각 조건에서 수행 시간에 차이가 유의하게 나타났다. 화살표 형태 feedback이 제공되었을 때, 수행 시간과 별 형태의 feedback이 제공되 었을 때의 수행 시간을 비교한 결과, 제스처 수행 시간은 차 이는 통계적으로 유의한 수준 $(p<0.001)$ 으로 나타났다. 또 별과 애니메이션의 경우도 두 피드백 간 제스처 수행 시간 의 차이가 통계적으로 유의한 수준인 것으로 나타났다 $(p<$ 0.001). 화살표 형태와 애니메이션 형태의 쌍체 비교 결과 도 마찬가지로 $p$-value가 0.0001로 visual feedback 종 류에 따른 제스처 수행 시간에 통계적으로 유의한 수준의 차이가 있는 것으로 판명되었다. 따라서 3 가지 visual

Table 4. Summary of paired t-test

\begin{tabular}{c|c|c|c}
\hline & Paired & df & $p$-value \\
\hline \multirow{4}{*}{ Elapsed time } & Mouse - Star & 719 & $<\mathbf{0 . 0 0 1}$ \\
\cline { 2 - 4 } & Star - Animation & 719 & $<\mathbf{0 . 0 0 1}$ \\
\cline { 2 - 4 } & Mouse - Animation & 719 & $<\mathbf{0 . 0 0 1}$ \\
\hline \multirow{4}{*}{ Trace length } & Mouse - Star & 719 & $<\mathbf{0 . 0 0 1}$ \\
\cline { 2 - 4 } & Star - Animation & 719 & $\mathbf{0 . 0 0 1 2 3}$ \\
\cline { 2 - 4 } & Mouse - Animation & 719 & 0.371788 \\
\hline \multirow{4}{*}{ Motion speed } & Mouse - Star & 719 & $<\mathbf{0 . 0 0 1}$ \\
\cline { 2 - 4 } & Star - Animation & 719 & $<\mathbf{0 . 0 0 1}$ \\
\cline { 2 - 4 } & Mouse - Animation & 719 & $\mathbf{0 . 0 0 3 0 5}$ \\
\hline
\end{tabular}


feedback 모두에 대해서 제스처 수행 시간 차이가 있다고 말할 수 있다.

\subsection{Length of hand trace in performing gesture}

3가지의 visual feedback 형태(별, 애니메이션, 화살표) 에 따라 제스처 수행 시 손이 움직인 거리에 차이가 있는지 를 검증하기 위해서 각 visual feedback 별로 제스처 20개 에 대한 반복 수 3 번 총 60 회의 수행을 12 명의 실험참여자 가 실시하여, 총 720 개의 데이터가 수집되었다. 이 데이터를 사용하여 세 집단 간 분산분석을 실시하였다.

분산분석 결과 $p$-value가 0.27 로 3 가지 visual feedback 에 대해서 제스처 수행 거리의 차이는 통계적으로 유의하지 않은 것으로 나타났다. 이는 제스처 종류에 따라 손의 궤적 모양이 달라지고, 필연적으로 손이 움직이는 거리도 달라지 기 때문이라고 해석된다. 따라서 분산분석이 아닌 쌍체 비교 를 통하여 visual feedback의 영향을 살펴보았다. 쌍체 분석 결과는 Table 4에 요약되어 있다.

화살표 형태 visual feedback이 제공되었을 때, 제스처 수 행 시 손이 움직인 거리와 별 형태 feedback이 제공되었을 때, 손이 움직인 거리를 비교한 결과, $p$-value가 0.001로 화살표 형태의 feedback과 별 형태의 feedback의 제스처 수행 거리 차이는 통계적으로 유의한 것으로 나타났다. 또한 별 형태의 feedback과 애니메이션 형태의 feedback의 쌍 체 비교 결과도 $p$-value가 0.002로 두 feedback간 제스 처 수행 거리의 차이가 통계적으로 유의한 것으로 나타났 다. 반면에, 화살표 형태의 feedback과 애니메이션 형태의 feedback 두 집단간 쌍체 비교 결과는 $p$-value가 0.74로 제스처 수행 거리에 따른 차이가 통계적으로는 유의하지 않 은 것으로 나타났다.

\subsection{Motion speed in performing gesture}

3가지의 visual feedback 형태(별, 애니메이션, 화살표) 에 따라 제스처 수행 시 손이 움직인 속도에 차이가 있는지 를 검증하기 위해서 각 visual feedback 별로 제스처 20개 에 대한 반복 수 3 번 총 60 회의 수행을 12 명의 실험참여자 가 실시하여, 총 720 개의 데이터가 수집되었다. 이 데이터를 사용하여 세 집단 간 분산분석을 실시하였다.

분산분석 결과 $p$-value가 0.006로 3 가지 visual feedback에 대해서 제스처 수행 속력의 차이가 통계적으로 유 의한 것으로 나타났다. 좀 더 구체적으로 각각의 visual feedback 간 수행 속력 차이를 알아 보기 위해 별 형태 feedback, 애니메이션 형태 feedback, 화살표 형태 feedback 3 가지 중 2 개씩 짝을 지어 쌍체 비교를 실시하였다.
쌍체 분석 결과는 Table 4 에 요약되어 있다.

Table 3에서 보듯이 쌍체 비교의 결과, 화살표의 형태 visual feedback이 제공되었을 때의 제스처 수행 속도와 별 의 형태 feedback이 제공되었을 때의 제스처 수행 속도 간 에는 통계적으로 유의한 차이가 있는 것으로 나타났다 $(p<$ 0.001). 또 별 형태와 애니메이션 형태의 경우도 $p$-value 가 0.0003 로 두 visual feedback 집단 간 제스처 수행 속 도에 통계적으로 유의한 차이가 있는 것으로 판명되었다. 화살표 형태와 애니메이션 형태의 쌍체 비교 결과도 마찬가 지로 $p$-value가 0.006로 제스처 수행 속력에 통계적으로 유의한 차이가 존재하는 것으로 나타났다.

\section{Discussion}

본 연구는 동작 인식을 사용한 원거리 조작 상황에서 디스 플레이 상의 visual feedback에 따라 사용자의 제스처가 어 떻게 달라지는지를 분석하기 위하여 제스처 수행 시 손이 움 직인 거리(trace length), 제스처 수행 시간(elapsed time), 제스처 수행 속도(motion speed)를 수집하였다. 결과적으로 거리, 시간, 속도 모든 요소 측면에서 피드백 제공 방식 간 의 유의한 수준의 차이가 존재하였다. 손이 움직인 거리의 경우 평균적으로 화살표 > 애니메이션 > 별 순서로 그 크기 가 컸는데, 이는 제스처를 크게 수행한 것으로 해석할 수 있 다. 수행 시간의 경우는 평균적으로 화살표 > 애니메이션 > 별 순서로 수행 시간이 길었다. 마지막으로 속력의 경우는 평균적으로 별 > 애니메이션 > 화살표 순서로 빠른 것을 확 인할 수 있었다.

이러한 차이는 visual feedback의 크기와 연관이 있다고 볼 수 있다 visual feedback 크기는 가장 작은 화살표 모 양을 기준(100\%)으로 애니메이션 형태 feedback의 크기 는 $156 \%$ 정도였고, 별 형태 feedback의 크기는 $250 \%$ 였 다. Visual feedback 크기에 관한 사실과 원거리 한 손 조 작 상황에서 visual feedback이 제스처 수행에 미치는 영향 을 함께 생각해보면, visual feedback의 크기가 클수록 평균 적으로 적은 거리, 짧은 시간, 빠른 속도의 특징을 가지는 것 을 알 수 있다.

이 결과로 미루어 보아 본 연구는 적용될 수 있는 사례를 생각해보면, TV를 제스처로 조작하는 경우, 주어진 상황에 따라서 적합한 visual feedback을 설계하는데 활용될 수 있 다. 화살표 형태의 작은 visual feedback은 본 연구에 의해 작은 범위, 긴 시간, 느린 속도가 특징이라고 규명할 수 있고 별 형태의 큰 visual feedback은 본 연구에 의해 큰 범위, 짧은 시간, 빠른 속도가 특징이라고 할 수 있으므로, 소수의 
대안을 신속하게 선택해야 하는 경우에 효과적으로 활용될 수 있을 것이다.

마지막으로 애니메이션 형태의 visual feedback의 경우 선호도 조사와 의견 조사에 의하면 사람들이 애니메이션 효 과에 대해 흥미를 많이 느끼고 결과적으로 선호도가 높은 것 으로 나타났다. 또한 피실험자들 본인이 수행하는 제스처에 대한 정확한 궤적이 visual feedback을 통해 보여지기 때문 에 다른 feedback에 비해 신중하게 제스처를 하려고 노력했 다는 의견도 많이 있었다. 이런 점들로 미루어 보아 애니메 이션 형태의 visual feedback은 제스처 인식에 있어서 제스 처 수행 형태의 정확성이 요구되는 경우에 사용되는 것이 적 합하다고 예상된다.

\section{Acknowledgements}

This work was supported by 2011 Hongik University Research Fund.

\section{References}

Chang, M. S., Kwak, S. D. and Kang, S. M., Presentation control system using gesture recognition and sensor, Journal of Korean Institute of Intelligent Systems, 21(4), 481-486, 2011.

Do, J. H., Jang, H., Jung, S. H., Jung, J. and Bien, Z., "Soft Remote control System in the intelligent Sweet home", IEEE RSJ International Conference on Intelligent Robots and Systems, 3(pp. 2193-2198), Edmonton, Canada, 2005.

Kühnel, C., Westermann, T., Hemmert, F., Kratz, S., Muller, A. and Möller, S., I'm Home: Defining and evaluating a gesture set for smart-home control, International Journal of Human-Computer Studies, 69(11), 693-704, 2011.

Lee, J., Kim, J. H. and Kim, T. Y., Fingertip extraction and hand motion recognition method for augmented reality applications, Journal of Korea Multimedia Society, 13(2), 316-232, 2010.

Lim, J., Jo, C. and Kim, D., (2012). "Analysis on User Variability in Gesture Interaction", forthcoming in proceedings of the ICHIT2012.

Lim, J., Choi, J. \& Kim, Y., A study on the effect of pre-cue in simple reactions of control-on-display interface, Journal of the Ergonomics Society of Korea, 30(4), pp. 563-569, 2011.

Parhi, P., Karlson, A. K. and Bederson, B. B., "Target Size Study for One-handed Thumb Use on Small Touchscreen Devices", Proceedings of the $8^{\text {th }}$ conference on Human-computer interaction with mobile devices and services(MobileHCU '06), (pp. 203-210), New York. NY. 2006.

Park, Y. S. and Han, S. H., Toch key design for one-handed thumb interaction with a mobile phone: Effects of touch key size and touch key location, International Journal of Industrial Ergonomics, 40(1), 68-76, 2010.

Stern, H. I., Wachs, J. P. \& Edan, Y., Designing hand gesture vocabularies for natural interaction by combining psycho-physiological and recognition factors, International Journal of Semantic Computing, 2(1), 137-160, 2008.

Sun, X., Plocher, T. and Qu, W., "An Empirical Study on the Smallest Comfortable Button/Icon Size on Touch Screen" In A. Nuray(Ed), Usability and Internationalization. HCI and Culture, Springer, Berlin, Heidelberg, 615-621, 2007.

\section{Author listings}

Junho Kim: kevin.junho.kim@gmail.com

Position title: Undergraduate student, Department of Industrial Engineering, Hongik University

Areas of interest: User Experience Analysis

Ji Hyoun Lim: limjh@hongik.ac.kr

Highest degree: $\mathrm{Ph} . \mathrm{D}$., University of Michigan

Position title: Assistant Professor, Department of Industrial Engineering, Hongik University

Areas of interest: Computational Cognitive Modeling, User-Driven NPD

Sung Hyun Moon: shmoon@pplab.snu.ac.kr

Highest degree: Master, Seoul National University

Position title: Ph.D. Candidate, Department of Computer Science, Seoul National University

Areas of interest: Human-Computer interaction, Augmented Reality,

Computer game, Interactive Storytelling

Date Received : 2012-07-20

Date Revised :2012-07-27

Date Accepted : 2012-07-28 